\title{
Seasonal sensitivity of genotypes in the humid tropics and its application to chicken breeding
}

\author{
O. M. A. Jesuyon ${ }^{1}$ and S. O. Oseni ${ }^{2}$ \\ ${ }^{1}$ Animal Breeding and Genetics Unit, Department of Animal Production and Health, Federal University P.M.B. \\ 373 Oye-Ekiti, Ekiti State, Nigeria \\ ${ }^{2}$ Animal Breeding and Genetics, Department of Animal Sciences, Obafemi Awolowo University, \\ Ile-Ife, Osun State, Nigeria \\ Correspondence to: O. M. A. Jesuyon (dr.oluwatosinjesuyon14@gmail.com)
}

Received: 27 January 2015 - Revised: 22 May 2015 - Accepted: 5 June 2015 - Published: 14 July 2015

\begin{abstract}
The study was aimed at elucidating the effect of seasons, namely the early wet (EW), late wet (LW), early dry (ED) and late dry (LD) seasons, on genotype sensitivity, its magnitude and application for selection and management of chickens of Bovan Nera (BN) and ISA Brown (IB) origins. Breeding and hatching records from 1999 to 2008 were collected from CHI (Ajanla) Farms and hatchery records, Ibadan, Nigeria. Cock weight $(\mathrm{CW})$, hen weight $(\mathrm{HW})$, hen-house egg production (HHP), egg weight (EW), fertility of egg set (FES) and pullet day-old chicks (PDC) hatched were examined. ANOVA revealed that there was significant $(P<0.05)$ genotype $\times$ season interaction effect. This interaction was observed between genotypic values when compared between seasons within parameters. Within-season sensitivity parameters indicated that $\mathrm{BN}$ was more sensitive than IB in HW and FES for all seasons. In ED and LD seasons, sensitivity values were inconsistent in pattern with output levels of FES, HES and PDC hatched because of interaction between genotypes and seasons. Within the LW season, all sensitivity indices for genotypes were consistent in magnitude with productive and reproductive values. Therefore, a genotype's seasonal sensitivity indices could be utilized for direct antagonistic selection in LW season between genotypes in humid tropics.
\end{abstract}

\section{Introduction}

Every genotype demonstrates a reaction norm to the environment in which it is reared. The environment may be different farms, production systems, housing conditions, feeds, seasons, temperature, nutrition, topography or locations that are shared in common by all experimental units under study or in any group. These are usually treated as "treatments" in experimental design. According to Falconer and Mackay (1996), to quantify an environment, the mean performance of all genotypes in that particular environment is estimated; this is termed the environmental value. The mean of each genotype in each specific environment is already known. If our environment is different seasons of the year, then statistically, genotype sensitivity will be the regression of a genotype's own phenotypic value on the phenotypic mean of all genotypes in that season (seasonal value) (Falconer and
Mackay, 1996). Khan et al. (2006) showed a classical case of seasonal sensitivity. They reported that the local chicken strains of Bangladesh recorded the highest egg production of $52.78 \%$ in winter, followed by $40 \%$ in summer, $5 \%$ in spring and $2.22 \%$ in late autumn. Therefore, seasonal sensitivity is a component of genotype $\times$ environment interaction. Large differences in sensitivity among genotypes in various seasons may lead to either a change in scale of genotypes or a reversal of the order of merit among the genotypes $(G \times E)$. Sensitivity indices may also help to understand responses of genotypes to different seasons in an environment and thus give comparative values which could assist in ranking them for selection. When an environment enhances the expression of a character or trait, it is termed "good", but when it depresses the expression of the character, it is termed "bad" (Falconer and Mackay, 1996). In this regard, the humid tropical region of south-west Nigeria may be classified as an 
"inclement environment" for animal production because of its endemic nature for diseases such as Newcastle disease (ND), (ILRI/World Bank, 2013), coccidiosis, salmonellosis and infectious bursal disease/Gumboro (Sonaiya and Swan, 2004), as well as its depressive climatic conditions, which hinder optimal productivity of layer chickens. For instance, the ambient conditions as typified by temperature ranges of 25.24 to $26.37^{\circ} \mathrm{C}$ and $\mathrm{RH}$ of 79.53 to $82.00 \%$ across seasons could be on the upper threshold for optimal performance of IB and BN. In good environments, a high sensitivity of genotypes brings high performance, but in poor environments it decreases characters and brings about low performance. In this environment, low sensitivity could result in high performance. Therefore, a selection for low sensitivity by antagonistic selection will probably select for the best individuals and genotypes in the environment (Falconer and Mackay, 1996). This study aimed at investigating seasonal sensitivity of genotypes, nature and application for selecting between parent-stock (PS) chicken genotypes, namely Bovan Nera (BN) and ISA Brown (IB), under the humid environmental conditions of Ibadan in tropical south-west Nigeria.

The research objectives were as follows:

- To investigate whether seasonal sensitivity indices alone could be used to select between two or more genotypes either within season or across seasons.

- To find out in which of the four seasons selection could be conducted with a high level of accuracy.

- To find out which of the two genotypes is more productive and better adapted to the humid tropical environment of south-west Nigeria.

\section{Materials and methods}

Farm production and hatchery records on an average population of 3896 hens and 600 cocks in a ratio of $6: 1$ per batch on Bovan Nera (BN) and ISA Brown (IB) parent-stock chickens were obtained from CHI (Ajanla) Farms, Ibadan, Nigeria, using 24 batches of each hybrid covering 10 years (19992008). These include cock weight (CW), hen weight (HW), hen-house egg production (HHP), egg weight (EW), fertility of egg set (FES), hatchability of egg set (HES) and pullet day-old chicks (PDC) hatched. For the study, the data were partitioned into seasons, namely early wet (EW, April-July), late wet (LW, August-October), early dry (ED, NovemberJanuary) and late dry (LD, February-March). They were then subjected to descriptive statistics, ANOVA, $t$ tests, Duncan's multiple range test (under ANOVA for means comparison) and regression analysis $(P<0.05)$ using SAS (1999). Experimental design was that of randomized complete block, with treatment as strains and the block as seasons. Interaction was examined by factorial ANOVA (SAS, 1999), while genotypes' seasonal sensitivity indices (GSSI) were estimated with regression procedures of Microsoft Excel (Ex- cel, 2007) using the reaction models below (Falconer and Mackay, 1996):

Two approaches were used to calculate genotype seasonal sensitivity. The first was the within-season sensitivity index using the seasonal mean value, $X$, as in Eq. (1):

$y=a+b X+e$,

where $y$ is the reaction norm index (genotypes observed or measured or reaction value in season), $a$ is the intercept or genotypic constant for parameter and season, $b$ is the sensitivity coefficient for parameter and season, $X$ is the seasonal mean value (mean of a particular parameter of all genotypes in the season) and $e$ is the random error component.

The above equation was adjusted to estimate the betweenseason environmentally corrected sensitivity indices, using the environmental mean value, $X_{\mathrm{E}}$, as in Eq. (2). This second approach standardized the seasonal sensitivity indices and enabled comparison of parameters between genotypes and seasons in the environment:

$y=a+b X_{\mathrm{E}}+e$,

where $y$ is the reaction norm index (genotypes observed or measured or reaction value in season), $a$ is the intercept or genotypic constant for parameter and season and $b$ is the sensitivity index or coefficient for parameter and season. $X_{\mathrm{E}}$ is the environmental mean value; this was taken as the mean of a particular trait, of all genotypes, in all four seasons in the environment. $X_{\mathrm{E}}$ was the general effect of the environment on the particular parameter in all genotypes. $e$ is the random error component.

The effect of each season on sensitivity index of each parameter within genotype was computed with the relationship below:

seasonal effect on a parameter $=$

between seasons sensitivity-

environmental mean sensitivity $=\left|b s-Z_{\mathrm{E}}\right|$

where $b s$ is the between-season sensitivity and $Z_{\mathrm{E}}$ is the mean sensitivity for the environment, i.e. $[(\mathrm{EW}+\mathrm{LW}+\mathrm{ED}+\mathrm{LD})$ sensitivity values/4].

Both genotypes were bred and managed in the same seasons and with the same feeding regime and management but with many batches reared in different seasons. These batches were used as replicates because ANOVA indicated a lack of significant difference $(P<0.05)$ among batches.

\section{Results}

Seasonal weather parameters of the study area are presented in Table 1. There were significant differences between seasonal weather parameters within the experimental period covered by the data. This means that the seasons were statistically different $(P<0.05)$, clearly defined and distinguishable 
Table 1. Mean seasonal weather parameters of Ibadan (1999-2008).

\begin{tabular}{|c|c|c|c|c|}
\hline Season & E wet & L wet & E dry & L dry \\
\hline Periods & Apr-Jul & Aug-Oct & Nov-Jan & Feb-Mar \\
\hline Rainfall $\left(\mathrm{cm}^{-3}\right)$ & $174.08^{\mathrm{a}}$ & $174.43^{\mathrm{a}}$ & $11.01^{\mathrm{b}}$ & $41.29^{b}$ \\
\hline Sunshine hours & $8.95^{\mathrm{a}}$ & $6.17^{\mathrm{b}}$ & $8.27^{\mathrm{ab}}$ & $10.41^{\mathrm{a}}$ \\
\hline Wind speed $\left(\mathrm{km} \mathrm{h}^{-1}\right)$ & $2.78^{\mathrm{b}}$ & $2.10^{\mathrm{c}}$ & $2.26^{\mathrm{bc}}$ & $3.57^{\mathrm{a}}$ \\
\hline Temperature $\left({ }^{\circ} \mathrm{C}\right)$ & $26.37^{\mathrm{c}}$ & $25.24^{\mathrm{d}}$ & $26.99^{b}$ & $28.70^{\mathrm{a}}$ \\
\hline Relative humidity (\%) & $79.53^{\mathrm{a}}$ & $82.00^{\mathrm{a}}$ & $66.37^{\mathrm{b}}$ & $65.45^{\mathrm{b}}$ \\
\hline Rainy days month $^{-1}$ & $12^{\mathrm{b}}$ & $14^{\mathrm{a}}$ & $1^{\mathrm{d}}$ & $4^{\mathrm{c}}$ \\
\hline
\end{tabular}

Note: means across rows with different superscripts are significantly $(P<0.05)$ different; E: early; L: late.

from each other. The results in Table 1 further reveal that the late wet season (August to October) was the wettest month, with rainfall, humidity and number of rainy days per month of $174.43 \mathrm{~cm}^{-3}, 82 \%$ and 14 days, respectively. This period had the lowest sunshine hours, wind speed and atmospheric temperature of $16 \mathrm{~h}, 2.1 \mathrm{~km} \mathrm{~h}^{-1}$ and $25.24^{\circ} \mathrm{C}$, respectively. The other seasons - early wet, late dry and early dry - followed in decreasing order of rainfall pattern.

The mean body weight, productive and reproductive traits of BN and IB PS chickens in Ibadan are presented in Table 2. These results reveal that there was no significant difference $(P>0.05)$ between genotypes in cock weight within seasons, but there was significant difference $(P<0.05)$ in hen weight between genotypes within the late dry season. In productive (hen-house egg production, HHP, and egg weight, EW) and reproductive (fertility of egg set, FES; hatchability of egg set, HES; and pullet day-old chicks hatched, PDC) parameters, the early wet season produced the largest significant differences $(P<0.05)$ between genotypes. Superior performance was obtained in the IB genotype in all parameters. The best fertility and hatchability results $(86.23,73.13 \%$ and 89.45 , $73.88 \%$ ) were obtained in both genotypes in the late wet season for BN and IB, respectively. BN had better hen weight (1724.81 vs. $1549.83 \mathrm{~g}$ ), while IB had superior cock weight (2214.14 vs. 2226.63 g), HHP (62.73 vs. 69.08$)$, egg weight (56.05 vs. 58.23 g), FES (83.61 vs. 86.70), HES (70.35 vs. $70.86)$ and PDC (33.10 vs. 34.36). ANOVA showed that there was significant $(P<0.05)$ genotype $\times$ season interaction; this interaction was observed as genotypic values were compared across seasons at parameter levels. The IB genotype had superior reproductive values of FES, HES and PDC $(\%)$ in the late wet season ( 89.45 vs. $86.23,73.88$ vs. 73.13 and 35.74 vs. 34.56 ), while $\mathrm{BN}$ recorded superiority in the late dry season ( 84.57 vs. $84.20,70.36$ vs. 67.73 , and 33.02 vs. 32.44) respectively.

Results on within-season sensitivity indices of parameters in both genotypes are presented in Table 3 . The results show that $\mathrm{BN}$ recorded the higher sensitivity values of the two genotypes. These values indicate that the BN genotype was more sensitive to seasonal variations than IB in hen weight and FES. The table also shows that, within the early wet and late dry seasons, the IB genotype recorded slightly higher sensitivity values in HHP (0.99 vs. 1.01 and 0.98 vs. 1.02), egg weight ( 0.96 vs. 1.04 and 0.98 vs. 1.02$)$, HES (0.86 vs. 1.14 and 0.91 vs. 1.09$)$ and PDC (0.85 vs. 1.15 and 0.91 vs. 1.09). Significant differences $(P<0.05)$ were observed between genotypes within season in FES sensitivities in the early dry season. Within the early wet and late wet seasons, there were significant $(P<0.05)$ differences in seasonal sensitivity indices between the two genotypes in reproductive parameters of FES, HES and PDC.

The results of between-season sensitivity indices of both genotypes are shown in Table 4. This table shows figures corrected for the environment for comparison between seasons for each parameter. These indices were not consistent in magnitude with production and reproduction outputs. In all cases, the ratio of true to untrue cases was $1: 1$ in production and $1: 2$ in reproduction. This would translate to about 50.00 and $33.33 \%$ accuracy in selection between genotypes if it were based on these indices.

The result of the effect of seasons on the seasonal sensitivity of BN and IB corrected for the environment is presented in Table 5. The negative seasonal-effect values in Table 5 showed the effect of season below the environmental mean, while positive values implied the effect of season above the environmental mean on the genotypes in the region. The late wet season produced an optimum mean seasonal effect of 0.148 for BN and -0.020 for IB genotypes. This revealed that, in the late wet season, BN produced the highest PDC of $34.56 \%$ from a hen weight, HHP and egg weight of $1677.74 \mathrm{~g}, 65.56 \%$ and $56.68 \mathrm{~g}$, respectively. Within the same season, IB produced a PDC of $35.74 \%$ from a hen weight, HHP and egg weight of $1596.93 \mathrm{~g}, 69.38 \%$ and $35.74 \mathrm{~g}$, respectively.

\section{Discussion}

The most optimal body weight for reproduction was obtained by $\mathrm{BN}$ and IB in the late wet and early wet seasons, respectively, indicating that the IB genotype probably required a 
Table 2. Mean weight, productive and reproductive output of Bovan Nera and ISA Brown chickens in Ibadan.

\begin{tabular}{llllllll}
\hline Parameter & Genotype & $n$ & E wet & L wet & E dry & L dry & Mean \\
\hline Cock weight (g) & BN & 600 & 2176.83 & 2162.93 & 2115.09 & 2451.72 & 2214.14 \\
& IB & 600 & 2221.87 & 2112.91 & 2543.66 & 2098.98 & 2226.63 \\
\hline Hen weight (g) & BN & 3805 & $1671.00^{\mathrm{y}}$ & $1677.74^{\mathrm{y}}$ & $1669.11^{\mathrm{y}}$ & $1923.11^{\mathrm{ax}}$ & 1724.81 \\
& IB & 3896 & 1590.67 & 1596.93 & 1497.56 & $1514.67^{\mathrm{b}}$ & 1549.83 \\
\hline HHP (\%) & BN & & 60.77 & 65.57 & $63.23^{\mathrm{b}}$ & 61.45 & 62.73 \\
& IB & 66.74 & 69.38 & $72.92^{\mathrm{a}}$ & 67.34 & 69.08 \\
\hline Egg weight (g) & BN & & $56.20^{\mathrm{b}}$ & 56.68 & 56.66 & $54.71^{\mathrm{b}}$ & 56.05 \\
& IB & & $59.99^{\mathrm{a}}$ & 57.97 & 58.12 & $56.88^{\mathrm{a}}$ & 58.23 \\
\hline Fertility of egg set (\%) & BN & & $80.82^{\mathrm{b}}$ & $86.23^{\mathrm{b}}$ & 82.77 & 84.57 & 83.61 \\
& IB & & $88.87^{\mathrm{a}}$ & $89.45^{\mathrm{a}}$ & 84.47 & 84.20 & 86.70 \\
\hline Hatchability of egg set (\%) & BN & & $69.08^{\mathrm{b}}$ & 73.13 & 68.85 & 70.36 & 70.35 \\
& IB & & $73.59^{\mathrm{a}}$ & 73.88 & 68.32 & 67.73 & 70.86 \\
\hline Pullet DOC (\%) & BN & $32.58^{\mathrm{b}}$ & 34.56 & 32.46 & 33.02 & 33.10 \\
& IB & $36.06^{\mathrm{a}}$ & 35.74 & 33.25 & 32.44 & 34.36 \\
\hline
\end{tabular}

Note: HHP, hen-house production; DOC, day-old chicks; BN, Bovan Nera; IB, ISA Brown; E, early; L, late; $n$, average population size; ab superscripts indicate significant difference within season; xy superscripts indicate significant difference within parameter.

Table 3. Within-season relative sensitivity indices for Bovan Nera and ISA Brown genotypes classified by traits.

\begin{tabular}{llllll}
\hline Parameter & Genotype & E wet & L wet & E dry & L dry \\
\hline Cock weight & BN & 1.00 & 1.02 & 1.16 & 0.97 \\
& IB & 0.99 & 0.98 & 0.84 & 1.03 \\
\hline Hen weight & BN & 1.05 & 1.03 & 1.06 & 1.01 \\
& IB & 0.95 & 0.97 & 0.94 & 0.99 \\
\hline HHP & BN & 0.99 & 1.03 & 1.02 & 0.98 \\
& IB & 1.01 & 0.97 & 0.98 & 1.02 \\
\hline Egg weight & BN & 0.96 & 1.11 & 1.04 & 0.98 \\
& IB & 1.04 & 0.89 & 0.96 & 1.02 \\
\hline Fertility of egg set & BN & $1.40^{\mathrm{a}}$ & $1.46^{\mathrm{a}}$ & $1.36^{\mathrm{a}}$ & 0.90 \\
& IB & $0.60^{\mathrm{b}}$ & $0.54^{\mathrm{b}}$ & $0.64^{\mathrm{b}}$ & 1.10 \\
\hline Hatchability of egg set & $\mathrm{BN}$ & $0.86^{\mathrm{b}}$ & $1.38^{\mathrm{a}}$ & 0.97 & 0.91 \\
& IB & $1.14^{\mathrm{a}}$ & $0.63^{\mathrm{b}}$ & 1.08 & 1.09 \\
\hline Pullet DOC & $\mathrm{BN}$ & $0.85^{\mathrm{b}}$ & $1.37^{\mathrm{a}}$ & 0.92 & 0.91 \\
& IB & $1.15^{\mathrm{a}}$ & $0.63^{\mathrm{b}}$ & 1.08 & 1.09 \\
\hline
\end{tabular}

Note: paired values within the same season with different superscripts are significantly $(P<0.05)$ different. E, early; L, late; BN, Bovan Nera; IB, ISA Brown.

slightly cooler environment with lower humidity, with more sunshine hours, higher wind speed and higher temperature $\left(79.53 \%, 8.95 \mathrm{~h}, 2.78 \mathrm{~km} \mathrm{~h}^{-1}\right.$ and $\left.26.37^{\circ} \mathrm{C}\right)$ than the $\mathrm{BN}$ genotype $\left(82.00 \%, 6.17 \mathrm{~h}, 2.1 \mathrm{~km} \mathrm{~h}^{-1}\right.$ and $\left.25.24^{\circ} \mathrm{C}\right)$ for optimum performance. Table 2 also shows the superior performance of the IB genotype over BN within seasons in the region.
Results also show that not all sensitivity indices were consistent in magnitude with reproduction (Table 3 ). In contrast to this general finding, all genotypic sensitivity indices within the late wet season were consistent in magnitude with productive and reproductive values. It was expected that the less sensitive genotype of the two strains under study, in any parameter, should possess the higher values in production and reproduction. Jinks and Connolly (1973) and Fal- 
Table 4. Between-season environmentally corrected sensitivity indices for Bovan Nera and ISA Brown genotypes classified by traits.

\begin{tabular}{llllllc}
\hline Parameter & Genotype & E wet & L wet & E dry & L dry & Environmental mean $\left(Z_{\mathrm{E}}\right)$ \\
\hline Cock weight & BN & 0.94 & 1.05 & 1.48 & 1.12 & 1.088 \\
& IB & 0.80 & 0.91 & 1.22 & 1.12 & \\
\hline Hen weight & BN & 0.99 & 1.06 & 1.21 & 1.10 & 1.000 \\
& IB & 0.62 & 0.89 & 1.04 & 1.08 & 1.000 \\
\hline HHP & BN & 0.98 & 1.01 & 0.99 & 1.03 & 1.563 \\
& IB & 0.96 & 1.04 & 0.99 & 0.99 & \\
\hline Egg weight & BN & 1.45 & 1.95 & 1.93 & 0.93 & 2.396 \\
\hline Fertility of egg set & IB & 1.51 & 1.85 & 1.93 & 0.97 & 1.359 \\
& BN & 2.63 & $3.29^{\mathrm{a}}$ & 2.63 & 1.67 & \\
\hline Hatchability of egg set & BN & 1.08 & 1.31 & $0.84^{\mathrm{b}}$ & 1.59 & 1.196 \\
& IB & 1.53 & 1.09 & $1.60^{\mathrm{a}}$ & 1.84 & \\
\hline Pullet DOC & BN & 1.03 & 0.91 & $0.16^{\mathrm{b}}$ & $1.61^{\mathrm{b}}$ & \\
& IB & 1.41 & 0.97 & $1.56^{\mathrm{a}}$ & $1.93^{\mathrm{a}}$ & \\
\hline
\end{tabular}

Note: E, early; L, late; BN, Bovan Nera; IB, ISA Brown. Paired values within the same season with different superscripts are significantly $(P<0.05)$ different. $Z_{\mathrm{E}}=[(\mathrm{EW}+\mathrm{LW}+\mathrm{ED}+\mathrm{LD})$ sensitivity values $/ 4]$.

Table 5. Seasonal (sensitivity) effect on Bovan Nera and ISA Brown genotypes classified by traits.

\begin{tabular}{llrrrr}
\hline Parameter & Genotype & E wet & L wet & E dry & L dry \\
\hline Cock weight & BN & -0.150 & 0.034 & 0.390 & 0.030 \\
& IB & -0.290 & -0.180 & 0.130 & 0.097 \\
\hline Hen weight & BN & -0.005 & 0.058 & 0.211 & 0.103 \\
& IB & -0.381 & -0.106 & 0.036 & 0.082 \\
\hline HHP & BN & -0.020 & 0.006 & -0.014 & 0.026 \\
& IB & -0.025 & 0.037 & -0.006 & -0.008 \\
\hline Egg weight & BN & -0.127 & 0.383 & 0.370 & -0.632 \\
& IB & -0.052 & 0.286 & 0.367 & -0.592 \\
\hline Fertility of egg set & BN & 0.233 & 0.889 & 0.231 & 0.731 \\
& IB & -0.025 & 0.318 & -0.238 & -1.154 \\
\hline Hatchability of egg set & BN & -0.276 & -0.045 & -0.520 & 0.230 \\
& IB & 0.169 & -0.268 & 0.237 & 0.476 \\
\hline Pullet DOC & BN & -0.168 & -0.290 & -1.040 & 0.417 \\
& IB & 0.218 & -0.227 & 0.364 & 0.729 \\
\hline Mean seasonal effect on genotype & BN & -0.073 & 0.148 & -0.053 & 0.129 \\
& IB & -0.055 & -0.020 & 0.195 & -0.053 \\
\hline
\end{tabular}

Note: E, early; L, late; BN, Bovan Nera; IB, ISA Brown. Seasonal effect on each parameter is the seasonal mean value minus the environmental mean value, i.e. $\left|b s-Z_{\mathrm{E}}\right|$ calculated from Table 4.

coner (1989) reported that antagonistic selection (selecting upwards in a bad environment by choosing the genotype with lower mean sensitivity) decreases environmental sensitivity of the genotype, while synergistic selection (selecting downward in a bad environment by picking individual or genotype with high sensitivity) increases environmental sensitiv- ity. However, lower sensitivity indices did not yield higher productive and reproductive values in all seasons between genotypes. The ratio of true to untrue cases was $1: 1$ in productive and 2:1 in reproductive parameters. This means that an antagonistic selection conducted randomly using withinseason sensitivity indices could only be 50 and $66.67 \%$ ac- 


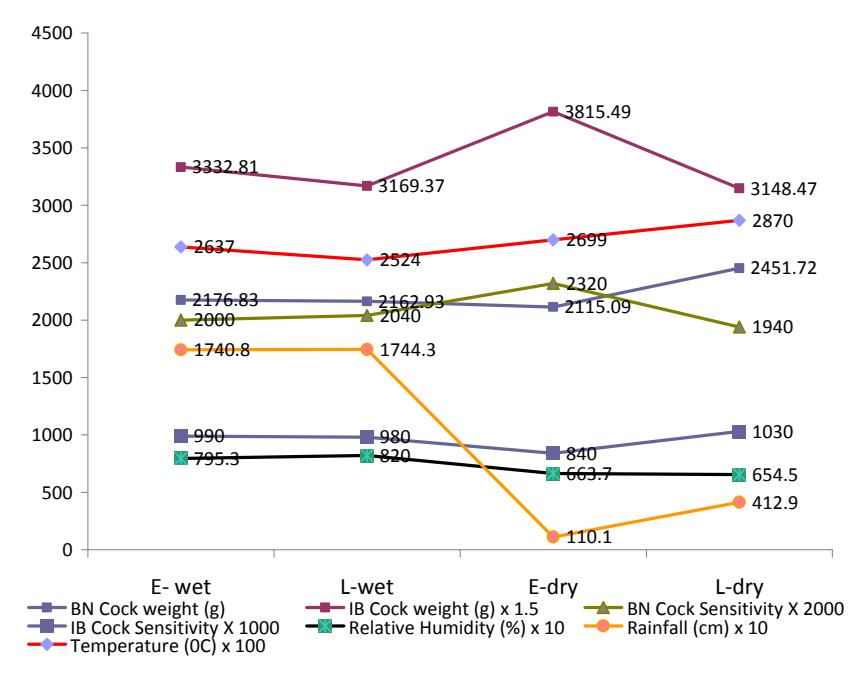

Figure 1. Graphs of cock weight and sensitivity indices for Bovan Nera and ISA Brown genotypes across seasons overlaid with temperature, rainfall and humidity parameters.

curate in productive and reproductive traits, respectively. In the experiments they reviewed, Jinks and Connolly (1973) and Falconer (1989) also found many exceptions to the rule. This lack of consistency in pattern of genotypes' seasonal sensitivity indices with production and reproduction values signified that randomly selecting between these two genotypes in any season of the year may not be appropriate within the environment; however this phenomenon itself could be due to continuous physiological adjustments and responses by genotypes to changing seasonal conditions.

We imply that selection conducted in the dry seasons for reproductive trait could yield an accuracy of about $33.33 \%$ only, based on the $1: 2$ ratio of true to untrue cases (Table 4). The between-season sensitivity result suggests that selection between genotypes could not be based on between-season values but instead on within-season values.

Results in Tables 2 and 3 suggest that results or outcomes of selection conducted within the late wet season and utilizing the within-season indices could be highly accurate because the less sensitive genotype had the higher production and reproduction figures. The results from these two tables thus suggest that the conduct of selection with genotypes' seasonal sensitivity indices in the late wet season between August and October could yield positive responses in the region. Study of these sensitivity indices also indicated interaction between genotypes and seasons in productive (HHP and egg weight) and reproductive parameters (FES and HES). In HHP, the BN genotype had superior indices in the late dry and early wet seasons, while the IB genotype had higher indices in the late wet and early dry seasons. In egg weight, however, BN recorded higher indices in the late wet season, while IB had better indices in the late dry and early wet seasons. In FES, BN hens showed greater sensitivity in the early wet, late wet and late dry seasons, while IB hens showed higher performance in the early dry season. The largest difference in sensitivity indices between the two genotypes was 0.58 in the late wet season, in favour of BN for FES. BN recorded higher sensitivity values in HES only in the late wet season, while IB hens had the upper values in the early wet, early dry and late dry seasons.

No interaction was observed between genotypes and seasons in hen weight sensitivity indices. While the hen weight of BN genotype was superior to that of IB in all seasons, the cock weight of IB was superior to that of $\mathrm{BN}$ in the early seasons, although the sensitivity index of IB was higher only in the late dry season, contrary to expectation - that is, following the trend from the early wet to late dry season, the sensitivity index of IB cock weight was expected to be lower in the late dry season also. This result could be due to the high environmental temperature and low humidity due to extreme conditions of the late dry season in the environment (Fig. 1). The interactions observed between seasons and genotypes in parameters revealed a re-ordering of the ranking of genotypes in different seasons of the year, thus suggesting the development of particular breeds for particular seasons, but since season is a random factor of the environment that is constantly changing, this effort might not yield a genotype with the best overall performance. Therefore a good result could be obtained between and within these two genotypes when selection was conducted in the late wet season whether reproductive values were utilized along with within-season late wet sensitivity indices or not. A seasonal atmospheric temperature of $25.24^{\circ} \mathrm{C}$ in combination with a RH of $82.00 \%$ could make an ideal condition for both genotypes for breeding and high productivity of layer chickens in a humid tropical environment like Ibadan. The case of the IB strain is worth mentioning. The highest PDC value of $36.06 \%$ was obtained in the early wet season from a hen weight, HHP and egg weight of $1590.67 \mathrm{~g}, 66.74 \%$ and $59.99 \mathrm{~g}$. The mean seasonal effect on the IB genotype in this season was -0.055 . This result suggests an optimum body weight range of 1590.67-1596.93 $\mathrm{g}$ for best performance of the IB genotype. For breeders, specific selection within the strain could be conducted on these two genotypes between April and October, when the atmospheric relative humidity (79.53-82.00\%) was higher and the atmospheric temperature $\left(26.37-25.24^{\circ} \mathrm{C}\right)$ was lower than that of other seasons of the year.

Kolmodin et al. (2003) showed in their study that environmental sensitivity increased in response to selection for high phenotypic value in the presence of $\mathrm{G} \times \mathrm{E}$ and an improving environment when reaction norms were linear or quadratic. Minderman et al. (2009) reported that individual differences in sensitivity to environmental cues are predicted to be part of animal personalities and are important because they will affect an individual's ability to respond to environmental change. Environmental sensitivity was measured in separate assays and was strongly predicted by what part of the environment was used during the preceding exploration be- 
haviour assays. It has also been reported that higher sensitivity in broilers results from the higher basal metabolic rate of broilers with more rapid growth rates (Mitchell and Sandercock, 1995; Sandercock et al., 1995; Cahaner et al., 1996). Fripp and Caten (1973) examined the relationship between the genetic systems determining mean expression and sensitivity to change in environment for dikaryotic growth rate in Schizophyllum commune. They concluded that the relationship between mean expression and sensitivity is markedly influenced by the environments involved, and that each combination of genotypes, environments and character should be treated as a separate case.

Jinks and Connolly (1973) showed that antagonistic selection reduces environmental sensitivity of genotypes while synergistic selection increases it. The experiments reviewed by these authors showed that sensitivity was lower after antagonistic selection than after synergistic. In other experiments reviewed, Falconer (1989) stated that antagonistic selection was significantly better than synergistic for changing the mean and suggested that antagonistic selection might be the best way to improve the mean performance in both good and harsh or unfavourable environments. Stabilizing selection refers to antagonistic selection in both good and bad environments on one genotype simultaneously. Kolmodin et al. (2002) investigated $\mathrm{G} \times \mathrm{E}$ interaction for production and fertility by use of a reaction norm model. Although significant genetic variation for the slope of the reaction norm was found, they found little re-ranking of sires, except between extreme environments. In agreement with their findings, the present study elicited a re-ranking of genotypes between early wet (IB) and late dry (BN) seasons. Hammami et al. (1992), in a cross-country environmental analysis of cattle, showed that estimates of genetic variance in the high, medium, and low classes of Tunisian environments were 45,69 and $81 \%$ lower, respectively, than the estimate found in the high management level in Luxembourg. They concluded that breeding decisions in a lowinput Tunisian environment should utilize semen from sires with daughters in similar production environments rather than semen of bulls proven in higher management levels. Cardoso and Tempelman (2012) concluded that the postweaning body weight gain (PWG) environmental sensitivity of imported North American-origin bulls $(0.046 \pm 0.009)$ was significantly larger $(P<0.05)$ than that of local sires $(0.012 \pm 0.013)$. Moreover, PWG of progeny of imported sires exceeded that of native sires in medium and higher production levels. On the other hand, Angus cattle locally selected in Brazil tended to be more robust to environmental changes and hence more suitable when production environments for potential progeny are uncertain. Abdou et al. (1977) studied the sensitivity of inbred lines of Fayoumi chicks to seasonal variations of winter and summer for fertility, hatchability, viability and body weight. They found out that inbred lines were more sensitive to seasonal variations than control lines, while the sensitivity of inbred lines de- creased as they advanced in age. The results of this study conformed to a previous report (Minderman et al., 2009) in that the sensitivity of parameters was influenced by the different seasons and this was considered as properties of behavioural and adaptive traits of genotypes in this study. Hau (2001) showed that seasonal breeding in a near-equatorial habitat is probably made possible by possession of an exceptionally high sensitivity to the slight seasonal changes in photoperiod at that latitude (maximal annual day length change of about $1 \mathrm{~h}$ ), and concluded that response to photoperiodic cues enabled birds to physiologically prepare for the coming breeding season ahead of time.

Recently, studies were performed on genotypeenvironment interaction for body weight adjusted to 205 days (Ambrosini et al., 2014b), for body weight adjusted to 550 days of age (Ambrosini et al., 2014a) and for weaning weight (Ribeiro et al., 2015) in Polled Nellore cattle. They showed in their reports that animals with higher genetic values respond better to environmental improvement. The existence of $\mathrm{G} \times \mathrm{E}$ was confirmed on body and weaning weight, emphasizing the need for specific evaluations for low-, medium- and high-level production environments. Ribeiro et al. (2015) emphasized the importance of considering $\mathrm{G} \times \mathrm{E}$ in the genetic evaluation of Nellore cattle, and that this phenomenon will result in increased selection efficiency in the weaning period.

To determine the better genotype in both seasons in the environment (that is, the genotype that will produce the better overall performance within both unfavourable and good seasons), one could compute the mean environmental effect (all seasons and all parameters) on each genotype (Table 5). This gave values -0.506 and 0.067 for $\mathrm{BN}$ and IB, respectively. The closer of these two values to the environmental mean value of 1.00 was that of the IB strain (0.067). The other approach is to measure performance over the four seasons, which in reality were random and uncontrollable environments, and then calculate the mean performance. Thus the results in Table 2 provide a solution - the IB strain was better over the four seasons, with higher mean values in all parameters except hen weight. A third approach is to estimate overall performance across the maximum threshold of all ambient conditions, including temperature and RH. A superior genotype could indicate higher adaptability and performance under maximum threshold of ambient conditions across all seasons. Using this criterion (Tables 1, 2 and Fig. 1), the BN strain was better in adaptability, but IB was superior in productivity across the seasons.

\section{Summary}

In selecting between genotypes or within genotypes, sensitivity values could consistute a very useful selection tool within the late wet season. However, while selecting between genotypes in the presence of genotype-season interaction, 
all-season mean values of parameters and sensitivity indices could be useful for antagonistic selection.

Author contributions. O. M. A. Jesuyon conceived the study, designed the experiments, did coordination and statistical analysis, and drafted the manuscript. S. O. Oseni participated in the interpretation of the results and drafting of the manuscript. Both authors read and approved the final manuscript.

Edited by: K. Wimmers

Reviewed by: G. Copur and one anonymous referee

\section{References}

Abdou, F. H., Soltan, M., Abd-Ellatif, M., and Ayoub, H.: Sensitivity of inbred Fayoumi chicks to seasonal variations, Ann. Genet. Sel. Anim., 9, 423-429, 1977.

Ambrosini, D. P., Carneiro, P. L. S., Neto, J. B., Filho, R. M., dos Santos Amaral, R., Cardoso, F. F., and Malhado, C. H. M.: Reaction norms models in the adjusted weight at 550 days of age for Polled Nellore cattle in Northeast Brazil, Rev. Bras. Zootecn., 43, 351-357, 2014a.

Ambrosini, D. P., Malhado, C. H. M., Neto, J. B., Filho, R. M., de Mello Affonso, P. R. A., and Carneiro, P. L. S.: Reaction norms of direct and maternal effects for weight at 205 days in Polled Nellore cattle in north-eastern Brazil, Arch. Tierzucht, 57, 1-11, doi:10.7482/0003-9438-57-032, 2014b.

Cahaner, A., Deeb, N., and Settar, P.: The Association between Broiler Potential Growth Rate and Sensitivity to Heat Stress, 45th Annual National Breeders Roundtable Proceedings, St. Loise, Missouri, USA, 29-41, available at: http://www. poultryscience.org/docs/pba/1952-2003/1996/1996Cahaner.pdf (last access: June 2015), 1996.

Cardoso, F. F. and Tempelman, R. J.: Linear reaction norm models for genetic merit prediction of Angus cattle under genotype by environment interaction, J. Anim. Sci., 90, 2130-2141, doi:10.2527/jas.2011-4333, 2012.

Falconer, D. S.: Introduction to Quantitative Genetics, edited by: Hill, W. G., 1989, in: Falconer, D. S: Selection in different environments: effects on environmental sensitivity (reaction norm) and on mean performance, Genet. Res., 56, 57-70, 1990.

Falconer, D. S. and Mackay, T. F. C.: Environmental Sensitivity, in: Introduction to Quantitative Genetics, 4th edition, Essex, Longman, 345, 133-134, 324-325, 1996.

Fripp, Y. J. and Caten, C. E.: Genotype-environmental interactions in Schizophyllum commune III, The relationship between mean expression and sensitivity to change in environment, Heredity, 30, 341-349, doi:10.1038/hdy.1973.43, 1973.
Hammami, H., Rekik, B., Bastin, C., Soyeurt, H., Bormann, J., Stoll, J., and Gengler, N.: Environmental sensitivity for milk yield in Luxembourg and Tunisian Holsteins by herd management level, J. Dairy Sci., 92, 4604-4612, doi:10.3168/jds.2008$1513,1992$.

Hau, M.: Timing of Breeding in Variable Environments: Tropical Birds as Model Systems, Horm. Behav., 40, 281-290, 2001.

ILRI/World Bank: World Bank Report No.: 75068, Nigeria Integrated animal and human health management project Final report, Disease burden and cost-benefit analysis of targeted interventions, 72 pp., www.researchgate.net/profile/John_Ihedioha/ publication (last access: June 2015), 2013.

Jinks, J. L. and Connolly, V.: Selection for specific and general response to environmental differences, Heredity, 30, 33-40, doi:10.1038/hdy.1973.4, 1973.

Khan, H., Miah, M. Y., Bhuiyan, M. M., Begum S., Hussein, M. M., and Khanunv, R.: Homestead Poultry Production in Sylhet region, International Journal of Poultry Science, 5, 1092-1095, www.pjbs.org/ijps/fin745.pdf (last access: June 2015), 2006.

Kolmodin, R., Strandberg, E., Jorjani, H., and Danell, B.: Selection in the presence of a genotype by environment interaction response in environmental sensitivity, Anim. Sci., 76, 375-385, 2003.

Kolmodin, R., Strandberg, E., Madsen, P., Jensen, J., and Jorjani, H.: Genotype By Environment Interaction in Nordic Dairy Cattle Studied Using Reaction Norms, Acta Agr. Scand. A-An., 52, 1124, 2002.

Microsoft Excel computer software: Regression Procedures, Microsoft Corporation, USA, 2007.

Minderman, J., Reid, J. M., Evans, P. G. H., and Whittingham, M. J.: Personality traits in wild starlings: exploration behavior and environmental sensitivity, Behav. Ecol., 20, 830-837, doi:10.1093/beheco/arp067, 2009.

Mitchell, M. A. and Sandercock, D. A.: Increased hyperthermia induced skeletal muscle damage in fast growing broiler chickens?, Poultry Sci., 74 (Supplement 1), 74, 1995.

Ribeiro, S., Eler, J. P., Pedrosa, V. B., Rosa, G. J. M., Ferraz, J. B. S., and Balieiro, J. C. C.: Genotype-environment interaction for weaning weight in Nellore cattle using reaction norm analysis, Elsevier B.V., 176, 40-46, doi:10.1016/j.livsci.2015.03.032, 2015.

Sandercock, D. A., Mitchell, M. A., and MacLeod, M. G.: Metabolic heat production in fast and slow growing broiler chickens during acute heat stress, Brit. Poultry Sci., 36, 868, 1995.

SAS/STAT Statistical Analytical Systems Computer software, SAS Institute Incorporated, N.C., USA, 1999.

Sonaiya, E. B. and Swan, S. E. J.: Manual of small-scale poultry production, Technical guide, Food and Agriculture Organization of the United Nations, Rome, Italy, www.fao.org/3/a-y5169e.pdf (last access: June 2015), 2004. 